\title{
POTENCIALIDADES ANTIMICROBIANAS DE LA GOSSYPITRINA AISLADA DE LAS FLORES DE Talipariti elatum S.W Y EVALUACIÓN DE ALGUNOS PARÁMETROS FARMACOGNÓSTICOS DE LAS FLORES
}

\author{
ANTIMICROBIAL POTENCIALITY OF GOSSYPITRINE ISOLATED FROM THE \\ FLOWERS OF Talipariti elatum S.W AND SOME PHARMACOGNOSTIC STUDIES \\ OF THE FLOWERS.
}

\author{
CUÉLLAR-CUÉLLAR, ARMANDO ${ }^{* *}$ Ph.D., ROJAS HERNÁNDEZ, NIDIA M ${ }^{2}$ \\ Ph.D. \\ ${ }^{1}$ Profesor Departamento de Farmacia, Instituto de Farmacia y Alimentos, \\ Universidad de La Habana, Cuba. ${ }^{2}$ Profesora Departamento de \\ Microbiología, Facultad de Biología, Universidad de La Habana, Cuba.
}

${ }^{*}$ Correspondencia: mandyc@infomed.sld.cu

Recibido: 21-11- 2010; Aceptado: 07-05-2011.

\section{Resumen}

Las flores de Talipariti elatum S.W, se utilizan como antiasmáticas en la medicina tradicional en Cuba. Nuestro grupo de trabajo ha demostrado la presencia de diferentes componentes químicos en ellas y en particular la estructura del gluco flavonoide Gossypitrina con altos rendimientos. Como continuación de estas investigaciones, en el presente trabajo se informan los resultados de algunos parámetros farmacognósticos para la posible calidad de las flores de esta planta como materia prima para su uso alternativo en medicina, así como la evaluación antimicrobiana de este flavonoide frente a cepas de diferentes microorganismos. Se evaluaron 13 cepas del género Candida y 26 cepas de otros microorganismos (19 Gram negativas y 7 Gram positivas).De ellas, se inhibieron en total 19 para un 53,85\% con valores promedio de Concentración Mínima Inhibitoria (CMI) de $29,6 \mathrm{mg} / \mathrm{mL}$ y de Concentración Mínima Bactericida (CMB) de 27,6 mg/mL, por lo que se puede considerar que la actividad antibacteriana de este flavonoide es apreciable, y vale la pena realizar un estudio más detallado de esta actividad para determinar su posible uso alternativo en tratamientos antibacterianos en humanos o en medicina veterinaria. No se presentó actividad frente a 13 cepas de levaduras del género Candida evaluadas.

Palabras clave: Talipariti elatum S.W., efecto antimicrobiano, gossypitrina.

\begin{abstract}
Talipariti elatum S.W flowers are widely used in Cuba as antiasthmatic in traditional medicine. Our group have described many chemical components from the extracts of the plant, in particular the flavonoid glucoside Gossypitrin in high yields. To continue the investigation with the plant, in this paper is summarized some quality control evaluations of the possible raw material for pharmaceutical use of the flowers and its extracts in human or veterinary
\end{abstract}


alternative medicine. The antimicrobial activity of Gossypitrin is undertaken with 26 strains of bacteria and 13 strains of Candida species. From 26 bacteria strains evaluated, (19 Gram negative and 7 Gram positive), 19 were inhibited (53, $85 \%$ ) with the average in concentration for Minimal Inhibitory Concentration (MIC) of 29, $6 \mathrm{mg} / \mathrm{mL}$ and for Minimal Bactericidal Concentration $(\mathrm{MBC})$ of $27,6 \mathrm{mg} / \mathrm{mL}$. Not antifungal activity against Candida strains was found. Those results suggest that Gossypitrin could be an interesting product to be evaluated as a possible alternative therapeutic agent for the treatment of infections in human or veterinary medicine.

Key words: Talipariti elatum S.W., antimicrobial activity, gossypitrin.

\section{Introducción}

La Gossypitrina es un flavonoide O-glucósido en la posición 7 de la estructura típica de un flavonoide polihidroxilado en las posiciones 3, 5, 8, 3' y 4 ' además de la posición 7 (Fig. 1). No es la única estructura presente en los extractos de los pétalos de las flores de Talipariti elatum $S$. $W$.

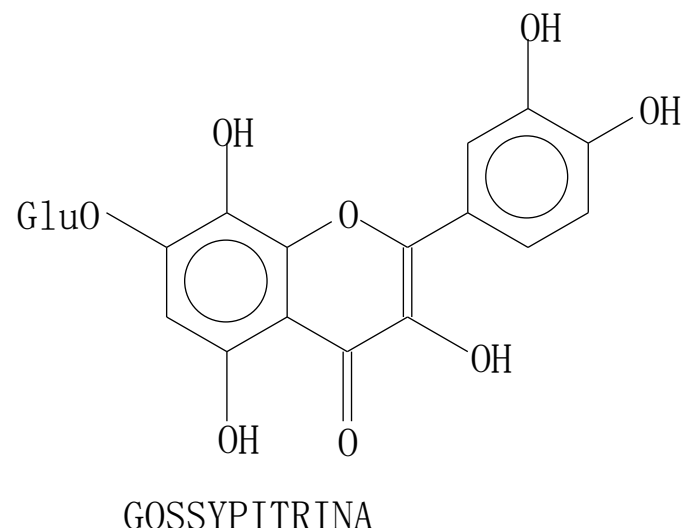

Figura 1. Estructura de Gossypitrina

Este compuesto se ha aislado de diversos tipos de flores, aunque en las especies del género Hibiscus es donde se ha encontrado su prevalencia de aparición junto con la Antocianidina roja que produce una asociación con este flavonoide que se conoce como efecto de copigmentación, por lo que generalmente aparecen asociados en los extractos de dichas flores. (DANGLES et al., 1993).

En la literatura consultada no existen informes anteriores sobre posibles efectos biológicos de esta molécula, solo los publicados por nuestro grupo de trabajo (PEREZ-TRUEBA et al., 2001; PEREZ-TRUEBA et al., 2003, CUÉLLAR et al., 2010,) y que se refieren a la evaluación de su posible efecto antioxidante 
por el método de la hepatotoxicidad inducida por el tetracloruro de carbono y la medición del malonaldehido producido en homogenato de cerebro de ratas. En particular, ni los extractos de las flores ni la Gossypitrina como tal se han evaluado en cuanto a su posible efecto antimicrobiano lo cual constituye el aspecto central de la presente investigación por el buen rendimiento obtenido de este flavonoide y por tanto la disponibilidad de cantidades suficientes de producto puro para las evaluaciones.

En Cuba hay dos especies de plantas conocidas por "majagua": Hibiscus tiliaceus, árbol pequeño y ramoso, y la majagua común o majagua azul, árbol que alcanza hasta cerca de $20 \mathrm{~m}$ de altura y que corresponde a Talipariti elatum S.W. especie considerada endémica y que hace un tiempo era clasificada como Hibiscus elatum S. W.

Sus flores en Cuba son muy apreciadas por la medicina tradicional como expectorante y antiasmática por ello el interés de comenzar las investigaciones con las mismas (ROIG, 1988).

La especie que crece en Cuba no tiene estudios químicos o biológicos, a no ser los de nuestro grupo de trabajo (MÁRQUEZ et al., 1999), que describan su composición en cuanto a metabolitos secundarios o que confirmen su posible uso, aunque si otras especies del género Hibiscus estudiadas, donde se describe la Gossypitrina como uno de los componentes principales de las flores (DANGLES et al., 1993).

Para su aislamiento en otras especies, se realiza una extracción de las flores completas con etanol, el residuo de extracción se reparte en disolventes tales como éter di etílico, cloroformo y acetato de etilo. La fracción de acetato de etilo se purifica en columnas de sílicagel con mezclas de acetato de etilo y metanol en un gradiente ascendente de polaridad, lo cual permite cristalizar entre otros, la Gossypitrina con rendimientos que oscilan alrededor del $0.1 \%$. (NAIR et al., 1961; SANKARA et al., 1961; LOWRY, 1976).

Talipariti elatum S.W. es un árbol muy común en toda la isla de Cuba, que florece generalmente en dos períodos del año. Sus flores inicialmente amarillas, cambian su color a naranja y rojo antes de caer de la planta. Estas flores completas, se utilizan por la población en forma de decocción o fermentadas en forma de un vino con fines expectorantes y antiasmáticos (ROIG, 1988). Las flores de la planta son las que se estudian en el presente trabajo para evaluar sus componentes químicos y su actividad antimicrobiana "in vitro" para su posible uso en medicina humana o veterinaria.

\section{Materiales y métodos}


Las flores de Talipariti elatum S. W. (Fig. 2) se colectaron en los alrededores del Instituto de Farmacia y Alimentos de la Universidad de la Habana en Mayo del 2009, separando las diferentes partes constitutivas antes del proceso de secado.

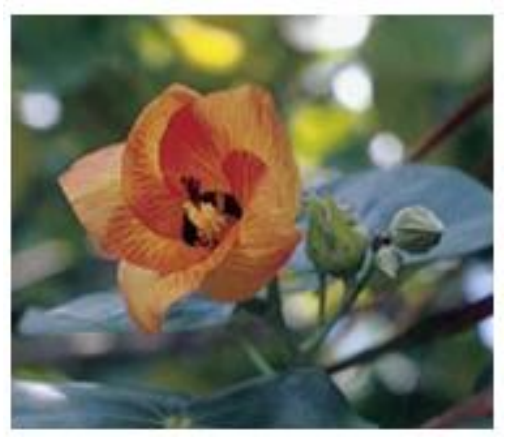

Figura 2. Flor de Talipariti elatum S.W.

La caracterización botánica se realizó en el Departamento de Sistemática del Jardín Botánico Nacional de Cuba, con una muestra para Herbario.

Los estudios farmacognósticos se realizaron de acuerdo a WHO/PHARM/ 80.50. Se evaluaron los siguientes parámetros de calidad de la materia prima vegetal: Secado en diferentes condiciones, determinación de humedad residual, determinación de cenizas, sólidos solubles extraibles en diferentes disolventes y un tamizaje fitoquímico general de acuerdo a (CUÉLLAR et al., 2010)

Extracción: Las diferentes partes de las flores de forma independiente (Cáliz, pétalos y pistilo con el polen), se someten a un proceso de extracción por disolventes sucesivos utilizando tolueno y etanol sobre el mismo material vegetal, de acuerdo con HOSTETTMANN et al. (1986). Para el aislamiento del flavonoide con el que se trabaja y evaluación de los parámetros farmacognósticos descritos, se utilizan de forma particular los pétalos separados de las otras partes de las flores por ser los que aportan los flavonoides objeto de las evaluaciones realizadas en la presente investigación.

Evaluación antimicrobiana: Para la evaluación antimicrobiana se utilizaron 19 cepas de bacterias Gram negativas y 7 cepas Gram positivas, así como 13 cepas de levaduras del género Candida. Todas fueron cepas de la colección ATCC, cuyos datos se registran en las tablas de los resultados para no duplicar información. La máxima concentración de Gossypitrina empleada para las determinaciones de CMI y CMB fue de $50 \mathrm{mg} / \mathrm{mL}$ en base a la solubilidad del flavonoide. Se utilizó Gentamicina y Nistatina como controles positivos de inhibición para los estudios microbiológicos realizados. 
La evaluación de la actividad antimicrobiana se realizó mediante el método de difusión en agar. Se prepara una capa fina de agar Nutriente estéril que se coloca en una placa Petri hasta su solidificación. Sobre esta capa se coloca una segunda capa de agar nutriente-inoculo que contiene una suspensión de cada cepa microbiana a evaluar. Una vez solidificadas ambas capas, se realizan cortes en el agar con un perforador estéril de $6 \mathrm{~mm}$ de diámetro y en el corte se coloca $100 \mu \mathrm{L}$ de las diferentes concentraciones de Gossypitrina a evaluar. Las placas se incuban por 24 horas a $37^{\circ} \mathrm{C}$. Para determinar las Concentraciones Mínimas Inhibitorias (CMI) se considera la menor concentración de este compuesto que forma un halo de inhibición del crecimiento en torno al corte de $10 \mathrm{~mm}$ de diámetro. La Concentración Mínima Bactericida $(\mathrm{CMB})$ se determina realizando pases o resiembras de cada tubo con las diferentes concentraciones del compuesto a evaluar en la superficie de placas con agar nutriente para determinar la presencia y realizar el conteo de colonias (BIAVATI et al., 2008). Todo el trabajo se realizó con tres repeticiones por cepa y concentración del compuesto.

\section{Resultados y discusión}

Estudio del secado: Dentro de las evaluaciones farmacognósticas usuales para poder utilizar un material vegetal se encuentra la evaluación del mejor método de secado que permite medir el mejor proceso y el menor tiempo para obtener un material de partida con la menor cantidad de agua que pueda interferir con los procesos de extracción o sobre la composición química del mismo si se necesita almacenarlo por algún tiempo.

En la Tabla 1 se muestran los resultados donde el método artificial en estufa permitió el secado en sólo tres días, la mayor cantidad en peso de pérdida de agua con un $73,5 \%$ y una humedad residual del $10,7 \%$ que es un valor que permite trabajar el material sin dificultades de acuerdo a lo establecido para plantas medicinales. Índices numéricos para la calidad de la materia prima:

Otros parámetros de calidad de la droga que se consideran útiles realizar después de secada la misma, son los que se relacionan a continuación con los siguientes resultados:

Las cenizas totales tuvieron un valor promedio de $10,5 \%$ este valor es ligeramente alto en comparación con los valores que se refieren para la mayoría de las plantas medicinales comerciales, pero cuando se realiza la diferenciación con la determinación de las cenizas solubles en agua el valor encontrado fue de $8,6 \%$ por lo que la diferencia es de $1,9 \%$ respecto al valor encontrado respecto a las cenizas totales lo cual asegura según lo establecido, que al estar dicho valor por debajo de un $3 \%$, no debe haber riesgos de tener grandes cantidades de metales pesados que puedan suministrar algún tipo de 
toxicidad a los extractos preparados o interferir en los resultados de la evaluación antimicrobiana encontrada al tener toxicidad sobre los microorganismos evaluados. Se determinaron además los sólidos solubles (extractivos) en agua y etanol, siendo los extractivos desde agua menores que los obtenidos desde el etanol (22,5\% vs $33,7 \%)$ lo cual asegura una mejor extracción en etanol de los componentes químicos de las flores.

Por su parte, el tamizaje fitoquímico de los extractos de agua y etanol mostraron los mismos resultados para los ensayos realizados:

Tabla 1. Resultado de las evaluaciones del secado de las flores.

\begin{tabular}{|l|l|l|l|}
\hline \multicolumn{1}{|c|}{ Método } & Días para secar & $\begin{array}{l}\text { Pérdida en peso } \\
\text { Por cada 100 g }\end{array}$ & Humedad residual (\%) \\
\hline Estufa $45^{\circ} \mathrm{C}$ & 3 & 73,5 & 10,7 \\
\hline Sombra & 18 & 65,0 & 19,8 \\
\hline Sol & 12 & 70,0 & 16,1 \\
\hline
\end{tabular}

Para los extractos de los pétalos: Ensayos positivos para: Flavonoides, antocianinas, compuestos reductores y aminas libres. Para los extractos del pistilo con polen: Ensayos positivos para: Compuestos reductores y aminas libres. Para los extractos del cáliz: Ensayos positivos para: Triterpenoides y esteroides, flavonoides, compuestos reductores y aminas libres.

Como se puede apreciar de estos resultados hay diferencias en los componentes químicos de las partes por lo que se justifica la necesidad de separar las mismas de acuerdo a lo que se desea que contengan los extractos.

Rendimiento de los diferentes extractos por partes evaluadas: En la Tabla 2 se presentan los rendimientos de los diferentes extractos obtenidos. Como puede apreciarse, el polen es el que aporta los mayores rendimientos, aunque en este caso son carbohidratos y derivados y aminas libres de forma fundamental como composición química de acuerdo a los análisis realizados por el sistema de Cromatografía Gaseosa acoplada a Masa, teniendo los pétalos un buen rendimiento, el cual corresponde principalmente a los flavonoides, que son las estructuras químicas que más interesan por los beneficios biológicos descritos para los mismos y por ser los componentes aislados y caracterizados de esta parte de la flor en nuestros estudios. 
Tabla 2. Rendimiento de sólidos totales por extracto y por las diferentes partes evaluadas.

\begin{tabular}{|l|c|c|}
\hline \multicolumn{1}{|c|}{ Partes de las flores } & $\begin{array}{c}\text { Extracto de tolueno } \\
\text { (\%) }\end{array}$ & $\begin{array}{c}\text { Extracto de etanol } \\
\text { (\%) }\end{array}$ \\
\hline Pétalos & 1,6 & 9,25 \\
\hline Polen & 6,06 & 20,0 \\
\hline Cáliz & 1,3 & 5,83 \\
\hline
\end{tabular}

Componentes químicos aislados y caracterizados de los diferentes extractos de los pétalos de las flores: Para los extractos de desengrase se caracterizaron fundamentalmente ácidos grasos por la técnica de Cromatografía Gaseosa acoplada a Masas, así como un alto porcentaje de componentes hidrocarbonados de cadenas largas de hasta 40 átomos de carbono y una sola estructura de núcleo esteroidal relacionada con el núcleo del estigmastano.

\section{Tolueno}

Hidrocarburos $>20 \%$ del peso del extracto total Glicerol, Ácido 3-4 dihidroxibenzoico, Ácido palmítico ( $\left.\mathrm{c}_{16: 1}\right)$, Ácido oleico ( $\left.\mathrm{c}_{18: 1}\right)$, Ácido esteárico $\left(\mathrm{c}_{18: 0}\right)$, Ácido graso $\left(\mathrm{c}_{23: 0}\right)$, Ácido graso $\left(\mathrm{c}_{25: 0}\right)$, Ácido graso ( $\left.\mathrm{c}_{27: 0}\right)$, Un esteroide con grupo carbonilo relacionado con el estigmasterol.

\section{Etanol}

En los extractos correspondientes al etanol se aislaron flavonoides, donde prevalece el glicósido Gossypitrina como componente de mayor rendimiento caracterizado por diferentes técnicas espectroscópicas ya publicadas (CUÉLLAR et al., 2010). Antocianina $>0.1 \%$ precipitada del extracto en medio ácido, Gossypitrina: 1,25\% de rendimiento a partir del material vegetal 13,5\% de rendimiento a partir del extracto.

\section{Actividad antimicrobiana del flavonoide Gossypitrina.}

En la Tabla 3 se muestran los resultados para las cepas de Salmonella y Shigella evaluadas. Se puede apreciar que estas cepas Gram negativas son bastante resistentes a la concentración evaluada siendo sólo una cepa de Salmonella inhibida y si fue inhibida la única cepa de Shigella ensayada, ambas a concentraciones inferiores a la de partida. 
Tabla 3. Valores de CMI y CMB de Gossypitrina para cepas de Salmonella y Shigella

\begin{tabular}{|l|c|c|c|}
\hline \multicolumn{1}{|c|}{ Cepa } & Resultado & CMI $\mathbf{~ m g} / \mathbf{m L}$ & CMB $\mathbf{~ g} / \mathbf{m L}$ \\
\hline $\begin{array}{l}\text { Salmonella enterica var Tiphy } \\
7251\end{array}$ & + & $\mathbf{3 5}$ & $\mathbf{3 2}$ \\
\hline $\begin{array}{l}\text { Salmonella enterica var } \\
\text { Typhimurium 14028 }\end{array}$ & - & $\mathbf{n o}$ & $\mathbf{n o}$ \\
\hline $\begin{array}{l}\text { Salmonella entérica var } \\
\text { Enteriditis 13076 }\end{array}$ & - & $\mathbf{n o}$ & $\mathbf{n o}$ \\
\hline $\begin{array}{l}\text { Salmonella enterica var Tiphy } \\
19430\end{array}$ & - & $\mathbf{n o}$ & no \\
\hline Shigella flexneri 12622 & + & $\mathbf{3 0}$ & $\mathbf{3 0}$ \\
\hline
\end{tabular}

En la Tabla 4 se muestran los resultados de cepas variadas, donde se observa que sólo las cepas de Bacillus, Providencia y Klebsiella resultaron inhibidas, teniendo la última cepa valores de CMI y CMB muy por debajo de la concentración inicial de partida, por lo que es muy sensible a este flavonoide.

Tabla 4. Valores de CMI y CMB de la Gossypitrina para distintas bacterias evaluadas

\begin{tabular}{|l|c|c|c|}
\hline \multicolumn{1}{|c|}{ Cepa } & Resultado & CMI $\mathbf{~ m g / m L}$ & CMB $\mathbf{~ m g / m L}$ \\
\hline Proteus mirabilis 7002 & - & - & - \\
\hline Bacillus subtilis 6633 & + & $\mathbf{5 0}$ & $\mathbf{5 0}$ \\
\hline Serratia marcescens 14056 & - & - & - \\
\hline Klebsiella pneumoniae 13883 & + & $\mathbf{1 0}$ & $\mathbf{1 0}$ \\
\hline $\begin{array}{l}\text { Providencia sp. } \\
\text { C-3450 }\end{array}$ & + & $\mathbf{4 0}$ & $\mathbf{4 0}$ \\
\hline Alcaligenes faecalis 1460 & - & - & - \\
\hline Streptococcus faecalis 29212 & - & - & - \\
\hline
\end{tabular}

En la Tabla 5 se muestran los resultados de la CMI y la CMB para las dos cepas de E. coli evaluadas, siendo sólo una de ellas inhibida a una concentración inferior de la inicialmente evaluada. Esta es una bacteria muy común en procesos infecciosos diversos, por lo que este resultado debe tenerse en cuenta para trabajos futuros con los flavonoides aislados de la planta.

Tabla 5. Valores de CMI y CMB de la Gossypitrina para cepas de E. coli

\begin{tabular}{|c|c|c|c|}
\hline Cepa & Resultado & CMI $\mathbf{~ m g} / \mathbf{m L}$ & CMB $\mathbf{~ m g / m L}$ \\
\hline E.coli 35150 & + & $\mathbf{2 0}$ & $\mathbf{2 0}$ \\
\hline E.coli 25922 & - & - & - \\
\hline
\end{tabular}


En la Tabla 6 se muestran los resultados para las dos cepas de Citrobacter, (Gram negativos) evaluados. Ambas cepas fueron inhibidas y sufrieron efectos letales por acción de la Gossypitrina, pero sólo una de ellas a concentraciones inferiores a la inicialmente utilizada de $50 \mathrm{mg} / \mathrm{mL}$.

Tabla 6. Valores de CMI y CMB de Gossypitrina para cepas de Citrobacter

\begin{tabular}{|l|c|c|c|}
\hline \multicolumn{1}{|c|}{ Cepa } & Resultado & CMI $\mathbf{~ m g / m L}$ & CMB $\mathbf{~ m g / m L}$ \\
\hline Citrobacter freundi 10625 & $\mathbf{+}$ & $\mathbf{3 0}$ & $\mathbf{3 0}$ \\
\hline Citrobacter freundi 8090 & + & $\mathbf{5 0}$ & $\mathbf{5 0}$ \\
\hline
\end{tabular}

De igual forma, en la Tabla 7 se muestran los resultados para las dos cepas de Proteus, (bacterias Gram negativas) evaluadas. De ellas, sólo una se inhibió a concentraciones muy inferiores a la inicialmente utilizada de $50 \mathrm{mg} / \mathrm{mL}$, por lo que en este caso los resultados son altamente apreciados. Sobre esa misma cepa (13315) la Gossypitrina presentó efecto bactericida.

Tabla 7. Valores de CMI y CMB de Gossypitrina para cepas de Proteus

\begin{tabular}{|l|c|c|c|}
\hline \multicolumn{1}{|c|}{ Cepa } & Resultado & CMI $\mathbf{~ g} / \mathbf{m L}$ & CMB $\mathbf{~ g ~} / \mathbf{m L}$ \\
\hline Proteus mirabilis 12453 & - & - & - \\
\hline Proteus vulgaris 13315 & + & $\mathbf{1 0}$ & $\mathbf{5}$ \\
\hline
\end{tabular}

En la Tabla 8 se muestran los resultados para las cepas de Staphylococcus evaluadas. Estas bacterias Gram positivas son una de las causas más frecuentes de infecciones y son los que han presentado mayor resistencia a los antimicrobianos comerciales, por lo que el hecho de que solo una cepa no fuera inhibida permite que se pueda evaluar este flavonoide con un posible antimicrobiano natural alternativo para hacer algunas valoraciones en infecciones in vivo debido a su baja toxicidad encontrada por nuestro grupo de trabajo.

Tabla 8. Valores de CMI y CMB de Gossypitrina para cepas de Staphylococcus

\begin{tabular}{|l|c|c|c|}
\hline \multicolumn{1}{|c|}{ Cepa } & Resultado & CMI $\mathbf{~ m g / m L}$ & CMB $\mathbf{~ m g / m L}$ \\
\hline S. aureus 6538 & - & - & - \\
\hline S. aureus 33862 & + & 20 & 20 \\
\hline S. aureus 25923 & + & 50 & 50 \\
\hline S. aureus clínico & + & 20 & 20 \\
\hline S. epidermidis 12228 & + & $\mathbf{5}$ & $\mathbf{5}$ \\
\hline
\end{tabular}


El último grupo de cepas evaluadas se muestran en la Tabla 9 para otras especies Gram positivas del género Enterococcus. Una sola cepa se inhibió a concentraciones inferiores a la inicialmente evaluada.

Tabla 9. Valores de CMI y CMB de Gossypitrina para cepas de Enterococcus

\begin{tabular}{|l|c|c|c|}
\hline \multicolumn{1}{|c|}{ Cepa } & Resultado & CMI $\mathbf{~ m g / m L}$ & CMB mg/mL \\
\hline Enterococcus cloacae 23353 & - & - & - \\
\hline Enterococcus aerogenes 13048 & - & - & - \\
\hline Enterococcus faecium 6056 & + & $\mathbf{3 0}$ & $\mathbf{3 0}$ \\
\hline
\end{tabular}

Se evaluaron 13 cepas del género Candida, pero ninguna resultó inhibida por el flavonoide.

En resumen, se puede concluir que de las 26 cepas de microorganismos evaluados, 19 bacterias Gram negativas y 7 Gram positivas, se inhibieron un total de 19 para un 53,85\%, con concentraciones promedios de CMI de 29,6 $\mathrm{mg} / \mathrm{mL}$ y de CMB de $27,6 \mathrm{mg} / \mathrm{mL}$, por lo que se puede considerar que la actividad antimicrobiana de este flavonoide frente a cepas de bacterias es apreciable y vale la pena realizar un estudio más detallado de esta actividad para determinar su posible uso alternativo en tratamientos antibacterianos en humanos o en medicina veterinaria ya que aunque los resultados están en todos los casos entre un 45-72 \% respecto a los controles positivos los flavonoides son estructuras químicas con menor toxicidad reconocida y pudieran ser una posible terapia alternativa a considerar. Se destaca que los controles positivos sólo se utilizan en el tamizaje inicial para seleccionar las cepas que se someten a la determinación de la CMl y la CMB y se seleccionan las cepas que tengan una inhibición no menor del $40 \%$ respecto al control positivo.

\section{Referencias}

BIAVATI, R.; PICEAGLIA, B.; MAROTTI, M. .2008 Antimicrobial activity of plant essential oils'. Dep. of Agro environmental Science and Technology University of Bologna. Disponible en: http://www.ienica.net/italyseminar/greenchem/biavatipresentation.pdf Consultado: 05.03-2008.

CUÉLLAR, A.; GONZÁLEZ, J. 2010. Obtención del glucósido flavonoide Gossypitrina de los pétalos de las flores de Talipariti elatum S. W. y evaluación de su posible efecto antioxidante. RECIA 2 (2) (En prensa)

DANGLES, D.; SAITO, N.; BROUILLARD, R. 1993. Copigment association of flavonoids with anthocianins in plants. Phytochemistry 34(1):119-124.

LOWRY, J.B. 1976. Floral anthocyanins of some Malasian Hibiscus species. Phytochemistry 15:1395-1396. 
MÁRQUEZ, I; CUELLAR, A; MARTÍNEZ, J. LORA, J. Y COL. 1999. "Estudio fitoquímico de la especie Hibiscus elatus S.w". Revista Cubana de Farmacia 33 (2):127-131.

NAIR A.G.R.; SUBRAMNIAN, S.; NAYARA-SWAMUN, S. 1961. Glycosides from flowers of Hibiscus tiliaceus. J. Sci. Ind. Research 20B:553-55.

hOSTETTMANN, K.; HOSTETTMANN, A.; MARSON, A. 1986 Preparative chromatography Techniques.
http://books.google.com.co/books?id=Y BnxeX-

cWgC\&printsec=frontcover\&dq=Preparative+chromatography+Techniques\&sou rce=bl\&ots=n8VPNXkhah\&sig=EHRelVbU2bVxS N59a4a551zQ5U\&hl=es\&ei= EWvpTKHMOsWBIAexpl2uCQ\&sa=X\&oi=book result\&ct=result\&resnum=1\&ve $\mathrm{d}=0 \mathrm{CB} 0 Q 6 A E w A A \# \mathrm{v}=0$ nepage \&q\&f=false. . Consultado: 12-10-2010.

PEREZ-TRUEBA, G.; RAMOS-GUANCHE, C.; MARTINEZ-SANCHEZ, B.; MARQUEZ-HERNANDEZ, I.; GIULIANI, A.; MARTINEZ-SANCHEZ, G. 2003. Protective effect of gossypitrin on carbon tetrachloride-induced in vivo hepatotoxicity. Redox Report 8(4):215-221.

PEREZ, T. G.; MARTINEZ, S.B.; MARQUEZ, H.I.; RAMOS, G.C. 2001. Evaluation of the antioxidant activity of gossypitrin by in vitro and in vivo assays. Revista Mexicana de Ciencias Farmacéuticas 32(2):19-24.

ROIG, J.T. 1988. Plantas Aromáticas, Venenosas y Medicinales de Cuba. Editorial Científico Técnica, Habana Cuba.

SANKARA, S.S.; NARAYAMA M.S. 1961. Pigments of the flowers of Hibiscus tiliaceus. J. Sci. Ind. Research 20B:133-134. 